\title{
A multi-threaded version of MCFM
}

\author{
John M. Campbell ${ }^{\mathrm{a}}$, R. Keith Ellis ${ }^{\mathrm{b}}$, Walter T. Giele ${ }^{\mathrm{c}}$ \\ Fermilab, PO Box 500, Batavia, IL 60510, USA
}

Received: 30 March 2015 / Accepted: 14 May 2015 / Published online: 3 June 2015

(C) The Author(s) 2015. This article is published with open access at Springerlink.com

\begin{abstract}
We report on our findings modifying MCFM using OpenMP to implement multi-threading. By using OpenMP, the modified MCFM will execute on any processor, automatically adjusting to the number of available threads. We modified the integration routine VEGAS to distribute the event evaluation over the threads, while combining all events at the end of every iteration to optimize the numerical integration. Special care has been taken that the results of the Monte Carlo integration are independent of the number of threads used, to facilitate the validation of the OpenMP version of MCFM.
\end{abstract}

\section{Overview}

An important aspect of Monte Carlo programs is evaluation speed and ease of use. A faster overall evaluation speed not only means that more complicated processes can be evaluated, but it also allows for more experimentation as results are returned in a shorter time.

Computer processors are increasing their computational power by including more and more computing cores. It is therefore essential for Monte Carlo event generators to explore the possibility of a parallel implementation of the code by taking advantage of the multiple threads to reduce the evaluation time for a given number of events. By properly implementing the use of multi-threading, the Monte Carlo evaluation speed will scale with the number of cores; this process will continue as more and more cores become available in the future. Monte Carlo event generators are well suited to take advantage of multi-core processors. Parallelization is straightforward as each generated event is evaluated independently, while the results of these evaluations are all combined to optimize the numerical integration.

\footnotetext{
a e-mail: johnmc@fnal.gov

be-mail: ellis@fnal.gov

c e-mail: giele@fnal.gov
}

The reason processors increase the number of cores instead of the processor frequency is the limitation deriving from the growth of the power consumption of the chip. The power consumption in a chip is given by the equation

$P=C V^{2} f$

where $P$ is power, $C$ is the capacitance being switched per clock cycle, $V$ is voltage, and $f$ is the processor frequency (cycles per second). As the clock speed increases the power (and hence heat) grows linearly. By having two circuits in parallel, we can double the capacitance and halve the clock speed. The voltage determines the rate at which the capacitance charges and discharges, so that a slower clock speed can run with lower voltages. At half the clock speed, we can approximately halve the voltage, leading to a saving in power without a compromise in performance. The use of many cores in this fashion may allow the growth of computing power to continue following Moore's law in the future. It is therefore imperative that software evolve to take advantage of these developments.

Currently the Intel Xeon-Phi coprocessor with 240 processor threads and General Purpose Graphics Processing Units (GPGPU's) with up to 2,880 gpu cores are the most extreme implementation of this approach to increasing the computational power. The Xeon Phi is the first generation of the Intel MIC (Many Integrated Cores) hardware. With an improved version of this coprocessor planned for release in the summer of 2015, further speed-ups can be expected.

We will explore using this co-processor and more conventional processors using OpenMP. Specifically, we will test our OpenMP version of MCFM ${ }^{1}$ on an Intel Core I7-4770 (4 hardware threads), a dual Intel Xeon X5650 ( $2 \times 6$ hardware threads), a quadruple AMD 6128 HE Opteron $(4 \times 8$ hardware threads) and the Intel Xeon-Phi 5110P (240 hardware

\footnotetext{
${ }_{1}$ MCFM-7.0 which runs under the OpenMP protocol as described in this paper can be downloaded from the mcfm.fnal.gov website.
} 
threads). Note that the Intel Core 17 comes with 8 hyperthreads, 2 software threads per core. The core can execute only one of the threads and quickly switch to the other thread if the current thread is waiting. As we will see this is of limited benefit for our application.

The OpenMP standard ${ }^{2}[1]$ is a good choice for implementing parallel programming. It is native to both the Intel and GNU compilers and can be invoked by including the 'openmp'-flag during compilation. No special libraries or other software need to be installed. The OpenMP compiler directives are simply implemented as comment statements in either FORTRAN or $\mathrm{C} / \mathrm{C}++$ code. This has the advantage that the code can be compiled without the 'openmp'-flag. In this case the OpenMP directives are interpreted as comments by the compiler. Furthermore, we can implement the parallelism with only minor alterations to the original code by just adding these compiler directives.

The further layout of our paper is as follows. In Sect. 2 we discuss some details and considerations for implementing OpenMP into the FORTRAN code of MCFM [2,3] (similar considerations will hold for $\mathrm{C} / \mathrm{C}++$ code). The numerical performance of the parallel code is explored in Sect. 3 using several different processors. Finally, in Sect. 4 we sum up our conclusions and review further possible developments for the OpenMP MCFM program.

\section{Implementing OpenMP in MCFM}

\subsection{MCFM}

MCFM is a parton level integrator, developed over many years at Fermilab, that calculates cross sections and distributions of kinematic variables for hard scattering processes in hadron-hadron collisions [2]. More than 300 processes are included, the majority of them calculated at next-toleading order in the strong coupling. The event generator consists of an adaptive integration routine which generates the events. The returned event probabilities are used to further optimize the integration using importance sampling. The program spends the bulk of its time in the event evaluation routines.

\footnotetext{
2 'OpenMP (Open Multi-Processing) is an API that supports multiplatform shared memory multiprocessing programming in $\mathrm{C}, \mathrm{C}++$, and Fortran, on most processor architectures and operating systems, including Solaris, AIX, HP-UX, Linux, Mac OS X, and Windows platforms. It consists of a set of compiler directives, library routines, and environment variables that influence run-time behavior. OpenMP is managed by the nonprofit technology consortium OpenMP Architecture Review Board (or OpenMP ARB), jointly defined by a group of major computer hardware and software vendors, including AMD, IBM, Intel, Cray, HP, Fujitsu, Nvidia, NEC, Red Hat, Texas Instruments, Oracle Corporation, and more.', from Wikipedia.
}

For MCFM the multi-dimensional integration is implemented using VEGAS [4,5]. It produces several iterations of sets of events. After each iteration the grid is optimized to reduce the weight fluctuations in the integration so that faster convergence is obtained. This offers an obvious and straightforward way to parallelize the program. While the grid optimization is not parallelized, so that all the results can be combined, the individual generation of phase space points and subsequent matrix element evaluation can be done in parallel as no data sharing is required between different events. This allows the parallel program to access all evaluated events to obtain maximum convergence, while the event evaluation is sped up considerably by using each thread for a different event generation and evaluation.

This should be contrasted with simultaneous running of an individual program on each thread. In this case the grids in each program are only updated with the events from that particular thread, leading to a worse convergence. The parallel version offers the advantage of combining the events from all threads for the grid optimization.

\subsection{OpenMP-MCFM}

Here we detail the work needed to produce an OpenMP implementation of MCFM. The MCFM code is large and complicated. To convert MCFM to an OpenMP supported MCFM requires some thought and work. We used as goals (a) to minimize the changes in the original code and (b) to implement the parallelism through comment compiler directives as much as possible. This makes the code compilable with or without the OpenMP flag. Another goal (c) was to make sure the program generates the same events independent of the number of threads used. We verified that the results obtained are independent of the number of threads used to evaluate the cross sections. This greatly helps to validate that the implementation of the parallel code is correct.

Almost all the work to be done is to make sure variables are correctly assigned. In a parallel program we have to decide whether a variable is global (i.e. potentially shared by threads) or local to the thread (i.e. each thread has its own version of the variable).

The most labor-intensive part is the treatment of data structures. The following rules will lead to a successful parallelization. For all the code running in parallel one has to implement the following steps:

- All variables in DATA statements in the parallel region have to be included in SAVE statements ensuring they are declared for each thread. If not done, the variables are not necessarily initialized.

- All variables in SAVE statements in the parallel region must be made 'thread private' in the respective functions and subroutines. 
- All common blocks whose variables are defined or changed in the parallel region have to be declared 'thread private' each time the common block is declared.

- All common blocks whose variables are defined or changed outside the parallel region in addition to being changed in the parallel region need to be declared 'thread private'. To ensure the values are copied to each thread at the start of the parallel region a COPYIN directive including the common block has to be issued.

Note that, where necessary, variables and common blocks are made 'thread private' by adding the THREADPRIVATE directive to the function or subroutine [1].

The MCFM code was originally written in FORTRAN 77, but parts of the code now require a FORTRAN 90 compiler. In view of the special treatment required for data statements, indicated above, it is beneficial to eliminate data statements wherever they are not needed. FORTRAN 90 allows parameter arrays, so it is useful to replace the FORTRAN 77 legacy data arrays by parameter arrays wherever possible.

To ensure that the same events are generated, independent of the number of threads used, we have to ensure VEGAS generates the same sequence of groups of pseudo-random numbers used to generate the momenta in an event. To do this we use the CRITICAL directive forcing the pseudorandom number generator to run serially, when assigning the groups of pseudo-random numbers to a thread. When looking at all threads combined, the same groups of random numbers will be generated, and consequently the same set of events. The order in which the groups of random numbers are accessed by the threads is not identical and varies from run to run (i.e. which thread reaches the critical region first) but in the end the same events are always generated. A named CRITICAL directive provides a way of distinguishing CRITICAL regions in different parts of the program. When a thread arrives at a CRITICAL directive, it waits until no other thread is executing a critical region with the same name.

The ATOMIC construct, which applies only to the specific assignment statement that follows it, can be an efficient alternative to a CRITICAL region. The statement following an ATOMIC directive is executed by all threads, but only one thread at a time can execute the statement.

This is still not sufficient to reach identical results for the cross section. The reason for this is numerical rounding differences due to the fact that the resulting weights are added in different orders. Using Kahan summation [6] will ameliorate rounding error, leading not only to identical cross section results but also to more accurate results.

We checked that all processes in MCFM produce identical results independent of the number of threads and in agreement with the non-parallel version of MCFM (version 6.8).

\section{Performance of OpenMP-MCFM}

\subsection{Runtime considerations}

We used version 3.0 of OpenMP to prepare our code, which includes all of the compiler directives discussed above. To compile the program the 'openmp'-flag has to be included. The resulting executable will use by default all available threads during execution. Note that if the program is compiled without the OpenMP flag it will not use multi-threading. To lower the number of threads used, two options are available. The first option uses the environmental variable OMP_NUM_THREADS. This variable can be set to the number of threads the OpenMP executable will use. Another possibility is to include the omp_lib.h library in the program which gives access to in-program OpenMP commands. The function call omp_set_num_threads(int) sets the number of threads used to the value of the integer 'int'. This allows for a dynamical change of the number of threads during execution. The library also gives access to many more OpenMP function calls, that are currently of no importance in running MCFM.

Another consideration is the memory stack size to be used by each thread. The default size of the stack is not specified by the OpenMP standard. If the stacksize is too small the program will crash with a segmentation fault or other unexpected behaviour. To be able to execute all processes in MCFM the stack size should be set to 16,000 or higher using the environmental variable OMP_STACKSIZE (though for most processes in MCFM a much smaller stacksize suffices).

\subsection{Results}

To benchmark the performance of the parallel version of MCFM we use four different types of computer hardware. This will test the code on a variety of hardware configurations with differing clock frequency, number of threads, cache size etc.

The first configuration is a standard desktop with an Intel Core i7-4770. This processor has 4 cores, each with 2 hyperthreads. The second configuration contains two Intel Xeon X5650 processors, each with 6 cores for a total of 12 cores. The third configuration contains four AMD 6128 HE Opteron processors, each with 8 cores for a total of 32 cores. The final configuration is an Intel Phi 5110P coprocessor card connected to a PCI slot. This coprocessor has 60 cores, each with 4 hardware threads for a total of 240 threads.

While we have validated all processes in this version of MCFM, we pick one process in particular to study the speedups gained by using multiple threads. The process we choose is $P P \rightarrow H(\rightarrow b \bar{b})+2$ jets which describes the production of a Higgs boson in association with two jets through an effective gluon-gluon-Higgs vertex. The Higgs boson subsequently undergoes a two-body decay to two $b$-quarks. Thus 
Table 1 The LO evaluation of $P P \rightarrow H(\rightarrow b \bar{b})+2$ jets using $4 \times$ $1000+10 \times 10,000$ Vegas events for the Intel Core I7-4770

\begin{tabular}{llllll}
\hline Thr. & \multicolumn{2}{l}{ Time $(\mathrm{s})$} & & $\begin{array}{c}\text { Acc. } \\
\text { Avg }\end{array}$ & Eff. (\%) \\
\cline { 2 - 5 } & Min & Avg & Max & & \\
\hline 1 & 1.67 & 1.69 & 1.70 & 1.00 & 100.00 \\
2 & 0.83 & 0.83 & 0.83 & 2.02 & 101.21 \\
3 & 0.57 & 0.57 & 0.58 & 2.94 & 97.88 \\
4 & 0.44 & 0.44 & 0.44 & 3.80 & 95.12 \\
5 & 0.40 & 0.40 & 0.40 & 4.18 & 83.50 \\
6 & 0.37 & 0.37 & 0.37 & 4.55 & 75.78 \\
7 & 0.34 & 0.34 & 0.34 & 4.92 & 70.26 \\
8 & 0.32 & 0.32 & 0.32 & 5.25 & 65.65 \\
\hline
\end{tabular}

Table 2 The LO evaluation of $P P \rightarrow H(\rightarrow b \bar{b})+2$ jets using $4 \times$ $1000+10 \times 10,000$ Vegas events for the Dual Intel Xeon X5650

\begin{tabular}{llllrr}
\hline Thr. & \multicolumn{2}{l}{ Time $(\mathrm{s})$} & & $\begin{array}{c}\text { Acc. } \\
\text { Avg }\end{array}$ & Eff. (\%) \\
\cline { 2 - 5 } & Min & Avg & Max & & \\
\hline 1 & 2.88 & 2.89 & 2.89 & 1.00 & 100.00 \\
2 & 1.49 & 1.49 & 1.50 & 1.94 & 96.76 \\
3 & 0.99 & 1.00 & 1.00 & 2.90 & 96.60 \\
4 & 0.75 & 0.75 & 0.75 & 3.85 & 96.13 \\
6 & 0.50 & 0.50 & 0.51 & 5.72 & 95.30 \\
8 & 0.38 & 0.38 & 0.38 & 7.57 & 94.59 \\
10 & 0.31 & 0.31 & 0.31 & 9.37 & 93.66 \\
12 & 0.26 & 0.26 & 0.26 & 11.16 & 92.96 \\
\hline
\end{tabular}

Table 3 The LO evaluation of $P P \rightarrow H(\rightarrow b \bar{b})+2$ jets using $4 \times$ $1000+10 \times 10,000$ Vegas events for the Quadruple AMD $6128 \mathrm{HE}$ Opteron

\begin{tabular}{llllrr}
\hline Thr. & \multicolumn{2}{l}{ Time $(\mathrm{s})$} & & \multicolumn{1}{l}{$\begin{array}{c}\text { Acc. } \\
\text { Avg }\end{array}$} \\
\cline { 2 - 5 } & Min & Avg & Max & Eff. (\%) \\
\hline 1 & 3.79 & 3.80 & 3.80 & 1.00 & 100.00 \\
2 & 2.00 & 2.02 & 2.05 & 1.88 & 94.06 \\
3 & 1.36 & 1.37 & 1.38 & 2.77 & 92.42 \\
4 & 1.03 & 1.04 & 1.05 & 3.66 & 91.52 \\
8 & 0.54 & 0.54 & 0.54 & 7.00 & 87.44 \\
12 & 0.38 & 0.38 & 0.38 & 9.98 & 83.13 \\
16 & 0.33 & 0.33 & 0.33 & 11.44 & 71.52 \\
32 & 0.83 & 0.84 & 0.86 & 4.50 & 14.06 \\
\hline
\end{tabular}

the process can have as many as 4 (5) jets in LO (NLO), two of which can come from the Higgs decay. In lowest order a process with $n$ particles in the final state requires $3 n-4$ phase space integrations and two integrals over parton density longitudinal fractions. Thus for this leading order (LO) process, a 10-dimensional integration is required. The next-to-leading (NLO) process requires a 13-dimensional integration. The results are contained in Tables 1, 2, 3 and 4 for the $\mathrm{LO}$ runs
Table 4 The LO evaluation of $P P \rightarrow H(\rightarrow b \bar{b})+2$ jets using $4 \times$ $1000+10 \times 10,000$ Vegas events for the Intel Xeon Phi 5110P

Intel Xeon Phi 5110P

\begin{tabular}{|c|c|c|c|c|c|}
\hline \multirow[t]{2}{*}{ Thr. } & \multicolumn{3}{|c|}{ Time (s) } & \multirow{2}{*}{$\begin{array}{l}\text { Acc. } \\
\text { Avg }\end{array}$} & \multirow[t]{2}{*}{ Eff. (\% } \\
\hline & Min & Avg & Max & & \\
\hline 1 & 23.09 & 23.12 & 23.15 & 1.00 & 100.00 \\
\hline 2 & 12.10 & 12.12 & 12.14 & 1.91 & 95.39 \\
\hline 3 & 8.14 & 8.22 & 8.53 & 2.81 & 93.78 \\
\hline 4 & 6.16 & 6.21 & 6.38 & 3.72 & 93.11 \\
\hline 16 & 1.66 & 1.67 & 1.68 & 13.86 & 86.61 \\
\hline 32 & 1.39 & 1.39 & 1.40 & 16.61 & 51.89 \\
\hline 64 & 1.41 & 1.41 & 1.41 & 16.39 & 25.61 \\
\hline 128 & 1.44 & 1.44 & 1.45 & 16.02 & 12.52 \\
\hline 240 & 1.52 & 1.52 & 1.53 & 15.19 & 6.33 \\
\hline
\end{tabular}

Table 5 The NLO evaluation of $P P \rightarrow H(\rightarrow b \bar{b})+2$ jets using $4 \times 1000+10 \times 10,000$ Vegas events for the Intel Core I7-4770

\begin{tabular}{|c|c|c|c|c|c|}
\hline \multirow[t]{2}{*}{ Thr. } & \multicolumn{3}{|c|}{ Time (s) } & \multirow{2}{*}{$\begin{array}{l}\text { Acc. } \\
\text { Avg }\end{array}$} & \multirow[t]{2}{*}{ Eff. (\%) } \\
\hline & Min & Avg & Max & & \\
\hline 1 & 238.83 & 238.95 & 239.07 & 1.00 & 100.00 \\
\hline 2 & 120.16 & 120.45 & 120.73 & 1.98 & 99.19 \\
\hline 3 & 81.99 & 82.03 & 82.07 & 2.91 & 97.10 \\
\hline 4 & 63.01 & 63.02 & 63.02 & 3.79 & 94.80 \\
\hline 5 & 58.67 & 58.69 & 58.71 & 4.07 & 81.43 \\
\hline 6 & 54.84 & 54.85 & 54.86 & 4.36 & 72.61 \\
\hline 7 & 51.52 & 51.53 & 51.54 & 4.64 & 66.24 \\
\hline 8 & 48.62 & 48.63 & 48.64 & 4.91 & 61.42 \\
\hline
\end{tabular}

Table 6 The NLO evaluation of $P P \rightarrow H(\rightarrow b \bar{b})+2$ jets using $4 \times 1000+10 \times 10,000$ Vegas events for the Dual Intel Xeon X5650

\begin{tabular}{lllllr}
\hline Thr. & \multicolumn{3}{l}{ Time $(\mathrm{s})$} & \multicolumn{2}{c}{ Acc. } \\
\cline { 2 - 4 } & Min & Avg & Max & Avg & \\
\hline 1 & 496.43 & 496.43 & 496.44 & 1.00 & 100.00 \\
2 & 249.73 & 249.83 & 249.94 & 1.99 & 99.35 \\
3 & 166.20 & 166.41 & 166.62 & 2.98 & 99.44 \\
4 & 124.58 & 124.58 & 124.59 & 3.98 & 99.62 \\
6 & 83.01 & 83.04 & 83.06 & 5.98 & 99.64 \\
8 & 62.24 & 62.26 & 62.29 & 7.97 & 99.66 \\
10 & 49.79 & 49.80 & 49.80 & 9.97 & 99.69 \\
12 & 41.46 & 41.46 & 41.46 & 11.97 & 99.78 \\
\hline
\end{tabular}

and in Tables 5, 6, 7 and 8 for the NLO runs. The tables contain, for each configuration and as a function of the number of threads used, the minimum, average and maximum runtime (in seconds), averaged over 10 runs for the first 3 configurations and 2 runs for the coprocessor. The acceleration compares the runtime to the single thread run time by taking the ratio of the two. Finally we give the efficiency in percent- 
Table 7 The NLO evaluation of $P P \rightarrow H(\rightarrow b \bar{b})+2$ jets using $4 \times 1000+10 \times 10,000$ Vegas events for the Quadruple AMD 6128 HE Opteron

\begin{tabular}{lllllr}
\hline Thr. & \multicolumn{2}{l}{ Time (s) } & & \multicolumn{1}{c}{ Acc. } & Eff. (\%) \\
\cline { 2 - 4 } & Min & Avg & Max & Avg & \\
\hline 1 & 806.86 & 806.98 & 807.10 & 1.00 & 100.00 \\
2 & 404.00 & 404.08 & 404.17 & 2.00 & 99.85 \\
3 & 269.26 & 269.37 & 269.48 & 3.00 & 99.86 \\
4 & 201.96 & 201.99 & 202.02 & 4.00 & 99.88 \\
8 & 101.03 & 101.05 & 101.07 & 7.99 & 99.83 \\
12 & 67.41 & 67.41 & 67.41 & 11.97 & 99.76 \\
16 & 50.56 & 50.56 & 50.56 & 15.96 & 99.75 \\
32 & 25.34 & 25.36 & 25.37 & 31.82 & 99.45 \\
\hline
\end{tabular}

Table 8 The NLO evaluation of $P P \rightarrow H(\rightarrow b \bar{b})+2$ jets using $4 \times 1000+10 \times 10,000$ Vegas events for the Intel Xeon Phi 5110P Intel Xeon Phi 5110P

\begin{tabular}{lrrrrr}
\hline Thr. & \multicolumn{2}{l}{ Time $(\mathrm{s})$} & \multicolumn{1}{l}{$\begin{array}{l}\text { Acc. } \\
\text { Avg }\end{array}$} \\
\cline { 2 - 4 } & \multicolumn{1}{l}{ Min } & \multicolumn{1}{l}{ Avg } & \multicolumn{1}{l}{ Max } & \multicolumn{1}{l}{ Avg } \\
\hline 1 & 3784.45 & 3784.45 & 3784.45 & 1.00 & 100.00 \\
2 & 1906.73 & 1906.73 & 1906.73 & 1.98 & 99.24 \\
3 & 1282.26 & 1282.26 & 1282.26 & 2.95 & 98.38 \\
4 & 958.59 & 958.59 & 958.59 & 3.95 & 98.70 \\
16 & 242.66 & 242.66 & 242.66 & 15.60 & 97.47 \\
32 & 121.25 & 121.25 & 121.25 & 31.21 & 97.54 \\
64 & 62.29 & 62.29 & 62.29 & 60.76 & 94.93 \\
128 & 41.22 & 41.22 & 41.22 & 91.81 & 71.73 \\
240 & 31.82 & 31.82 & 31.82 & 118.94 & 49.56 \\
\hline
\end{tabular}

ages. The efficiency is simply the acceleration divided by the number of threads. For a perfect parallelization, doubling the number of threads should double the acceleration, leaving the efficiency at $100 \%$. All the average runtime results of the tables are represented graphically in Fig. 1 where we plot on a log-log scale the runtime as a function of the number of threads used. Note that we do not generate histograms during these benchmarking runs.

We will first look at the results for the Intel Core i7 in Tables 1 and 5 (and Fig. 1). As we can see the speed-up as far as 4 threads is good, with an acceleration for LO up to 3.80 and for NLO up to 3.79. As the processor has 4 cores, each thread runs on a different core. If we use more than 4 threads some or all of the threads will share a single core with another thread. If one of the threads has to wait for a memory fetch, the core will switch to the other thread and start executing. As can be seen, this results in a much slower speed-up though some speedup is still achieved (from 3.80 for 4 threads to 5.25 for 8 threads at LO and from 3.79 to 4.91 at NLO). Yet, by using multi-threading on this basic configuration one can generate
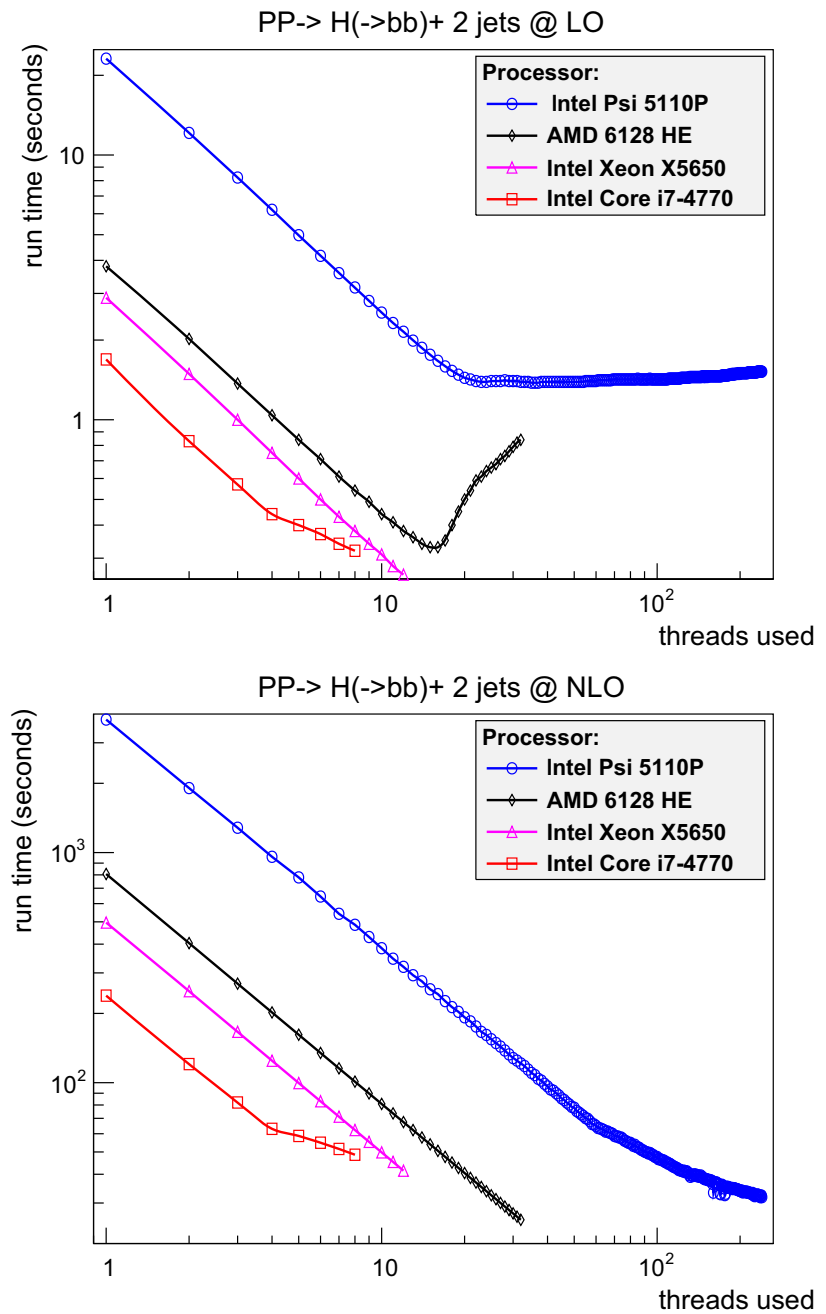

Fig. 1 The evaluation time of $P P \rightarrow H(\rightarrow b \bar{b})+2$ jets using $4 \times$ $1,000+10 \times 10,000$ Vegas events (in seconds) versus the number of threads. The top graph is at LO and the bottom graph at NLO

around 6.3 million Vegas events at NLO order per hour. Note that this depends on the cuts applied, as this will affect the number of rejected events. However, the comparison to other configurations is illuminating.

The next configuration to consider is the dual socketed X5650 processors giving a total of 12 cores. The results of Tables 2 and 6 show good scaling for LO with a maximum acceleration of 11.16. At NLO the acceleration is nearly perfect with a maximum acceleration of 11.97 using 12 threads. The difference in speed-up between LO and NLO can be understood by the fact that the NLO process is computationally bound (i.e. the runtime is predominantly determined by floating point operations), while at $\mathrm{LO}$ the computational component is much smaller and the memory fetch time will become more dominant, i.e. LO is more bandwidth bound. In other words at $\mathrm{LO}$ we do not give the cores enough floating point operations to keep them fully occupied. While this processor runs a factor of 0.56 slower than the Core i7, in the 


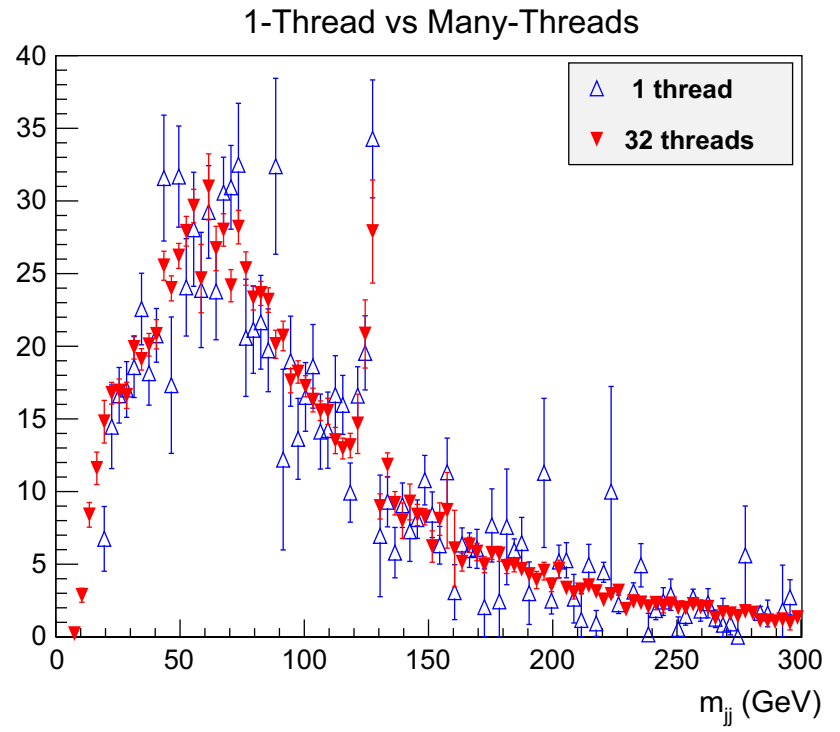

Fig. 2 The di-jet differential cross section for $P P \rightarrow H(\rightarrow b \bar{b})+2$ jets at NLO using $1 \mathrm{~h}$ of running time on the Intel Core I7-4770 using a single thread and on the quadruple AMD $6128 \mathrm{HE}$ Opteron using all 32 threads. The peak at $m_{j j}=125 \mathrm{GeV}$ when the two jets come from the decay of the Higgs boson is visible

end it wins out due to the use of 12 cores. By using multithreading on this configuration one can generate 8.7 million Vegas events at NLO order per hour.

In Tables 3 and 7 we move on to the quad-socketed AMD 6128 HE Opteron processors giving a total of 32 cores. We very clearly see the effect of the bandwidth bound LO and the computational bound NLO. The NLO gives nearly perfect acceleration of 31.82 , while $\mathrm{LO}$ reaches its maximum acceleration of 11.44 using 16 of the 32 cores. Using more than 16 cores actually makes the evaluation time slower as the bandwidth limitation becomes more important than the computational one. Despite being slower by a factor 0.35 compared to the Core I7 processor one can generate 14.2 million Vegas events at NLO per hour.

The final configuration is the Xeon-Phi coprocessor with 240 hardware cores. To achieve good acceleration it is crucial to have a computational bound calculation. This is dramatically demonstrated in Tables 6 and 8. At LO it achieves its fastest evaluation time using around 32 threads with an acceleration around 16.61. However at NLO the coprocessor keeps accelerating up to 240 threads for the evaluation time of $31.82 \mathrm{~s}$, giving a maximum acceleration of around 119. One can generate 10.7 million Vegas events at NLO per hour. While this co-processor has an impressive acceleration of over a factor of 100 , the processing speed of a single core is slow. (It is a factor of 0.07 slower than the Core i7). The next iteration of the co-processor is expected to be significantly faster, making this MIC architecture very attractive in the near future. It is worth noting this co-processor is a PCIbus card which, given the right configuration, can be added

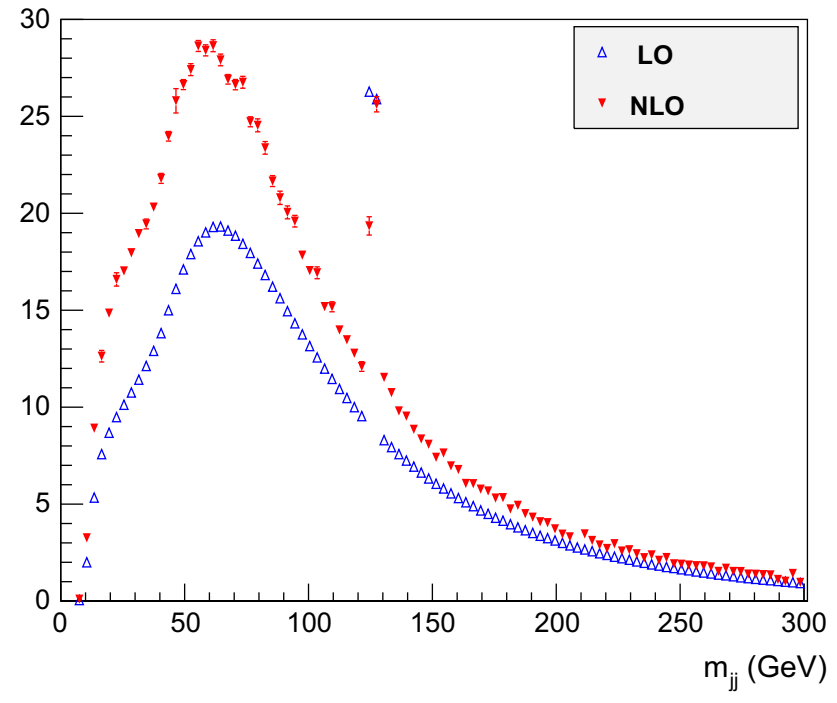

Fig. 3 The di-jet differential cross section for $P P \rightarrow H(\rightarrow b \bar{b})+2$ jets using $4 \times 1,500,000+10 \times 15,000,000$ events. At LO we use the Dual Intel Xeon X5650 with 12 threads (about 12 min of runtime) and at NLO we use the quadruple AMD $6128 \mathrm{HE}$ Opteron (about $22 \mathrm{~h}$ of runtime) with 32 threads. The peak when the two jets come from the decay of the Higgs boson is clearly visible

to a desktop turning it into a very powerful stand-alone event generator.

To see the impact of the faster running we show in Fig. 2 the results for the di-jet mass invariant mass distribution. We compare the fastest single thread configuration (the CoreI7) and the fastest multi-thread configuration (quad AMD) using approximately $1 \mathrm{~h}$ of runtime for each. We see that the single thread run is insufficient for any useful exploratory runs. In contrast one hour of running on the multi-threaded system gives a good result. Finally, in Fig. 3 we make the di-jet distribution using about $24 \mathrm{~h}$ of runtime which is more than sufficient to produce a stable final result.

\section{Conclusions}

To conclude we see that the threaded version of MCFM accelerates well on different architectures. The computationally bound NLO processes scale well with the number of threads and the evaluation speeds are significantly improved. In particular, the performance of the Xeon-Phi coprocessor is impressive. A new coprocessor is to be released in the summer of 2015, promising even faster evaluation times. Moreover, this new version will also be available in a socketed version, removing the PCI-bus and hopefully alleviating the bandwith-bound issues of LO. This will make the Xeon-Phi coprocessor a very attractive option for Monte Carlo generators in the near future.

As we have shown, we have successfully implemented a parallel version of MCFM. It instantly reduces the execution 
time dependent on the hardware configuration of the system (i.e. number of cores, cache configuration, memory bandwidth, clock frequency etc) without any intervention of the user of MCFM. For the computing intensive next-to-leading order processes we obtain very good accelerations on all processors. In particular, utilizing the Xeon-Phi coprocessor with 240 hardware cores yields an acceleration of order 100 over running on a single thread.

The new Xeon-Phi processor, to be released in summer 2015, will overcome most of the bandwith limitation to which the compute-light leading order processes are subject. Moreover the new processor will be substantially more powerful, giving us accelerations well over a factor of 100. Now that we have improved the speed of MCFM, we can implement more complicated processes in the event generator and still get acceptable evaluation times. Possibilities could include adding more jets to current processes in MCFM or proceeding to next-to-next-to leading order processes.

Acknowledgments The numerical work on the Intel Xeon-Phi processor was performed using the Fermilab MIC development cluster funded by the DOE Office of Science and operated by the Fermilab scientific computing HPC department. We acknowledge useful discussions with Don Holmgren and James Simone. This research is supported by the US DOE under contract DE-AC02-07CH11359.
Open Access This article is distributed under the terms of the Creative Commons Attribution 4.0 International License (http://creativecomm ons.org/licenses/by/4.0/), which permits unrestricted use, distribution, and reproduction in any medium, provided you give appropriate credit to the original author(s) and the source, provide a link to the Creative Commons license, and indicate if changes were made.

Funded by SCOAP $^{3}$.

\section{References}

1. B. Chapman, G. Jost, R. van der Pas, Using OpenMP (MIT press, Cambridge, 2007)

2. J.M. Campbell, R.K. Ellis, Nucl. Phys. Proc. Suppl. 205-206, 10 (2010). doi:10.1016/j.nuclphysbps.2010.08.011

3. J.M. Campbell, R.K. Ellis, C. Williams, JHEP 1107, 018 (2011). doi:10.1007/JHEP07(2011)018

4. G.P. Lepage, J. Comput. Phys. 27, 192 (1978). doi:10.1016/ 0021-9991(78)90004-9

5. W.H. Press, S.A. Teukolsky, W.T. Vetterling, B.P. Flannery, Numerical Recipes in FORTRAN: The Art of Scientific Computing (Cambridge University Press, Cambridge, 1992)

6. W. Kahan, Commun. ACM 8(1), 40 (1965). doi:10.1145/363707. 363723 